\title{
Modelo de regressão linear para estimativa de geração de RCD em obras de alvenaria estrutural
}

\author{
Linear regression model to estimate CDW generation in \\ structural masonry buildings
}

\section{Marcelo Oliveira Caetano \\ Asafe Bonisoni Fagundes Luciana Paulo Gomes}

\section{Resumo \\ A}

predição da geração de resíduos de construção e demolição (RCD), na fase de projeto, é etapa essencial do gerenciamento de resíduos do canteiro de obras. Esses índices podem contribuir para a redução de perdas e, consequentemente, redução de custos operacionais. Devido às dificuldades em executar uma correta estimativa dos RCD a serem gerados, o presente trabalho propôs um modelo de regressão linear múltipla para estimativa da geração de resíduos de obra em empreendimentos residenciais verticais com alvenaria estrutural. Obteve-se como resultado, para o modelo de regressão desenvolvido, um coeficiente de determinação $\left(\mathrm{R}^{2}\right)$ de 0,802 e coeficiente de determinação ajustado $\left(\mathrm{R}^{2}\right.$ ajustado) de 0,741 (intervalo de confiança de 95\%). A comparação entre os dados reais de geração de RCD e a estimativa gerada pelo modelo de predição desenvolvido por esta pesquisa mostrou erros na faixa de 10,62\% a 54,94\%. Assim, o modelo de regressão mostrou-se aplicável para os tipos construtivos viga-pilar e alvenaria estrutural. Pode ser utilizado por engenheiros e arquitetos, como uma ferramenta para estimativa de RCD no Plano de Gerenciamento de Resíduos de Construção e Demolição (PGRCD) da obra.

Palavras-chaves: Resíduos de construção e demolição. Modelo de regressão linear múltipla. Geração de RCD. Edificações verticais. Alvenaria estrutural.
Marcelo Oliveira Caetano Universidade do Vale do Rio dos Sinos São Leopoldo - RS - Brasil

Asafe Bonisoni Fagundes Universidade do Vale do Rio dos Sinos São Leopoldo - RS - Brasil

Luciana Paulo Gomes Universidade do Vale do Rio dos Sinos São Leopoldo - RS - Brasil

Recebido em 16/09/16 Aceito em 04/08/17

\begin{abstract}
The prediction of the construction and demolition waste (CDW) done during the design phase is essential to ensure proper waste management on construction sites. Similarly, these indices can contribute to reducing building losses and operating costs. Because of the difficulties to quantify CDW, this article proposes a multiple linear regression model to estimate the CDW generation in vertical residential buildings with structural masonry. Considering a confidence interval of 95\%, the coefficient model $\left(R^{2}\right)$ was calculated at 0.802 and the adjusted coefficient model (adjusted $R^{2}$ ) obtained was 0.741. The comparison between CWD generation measured on the building site and the estimate produced by the prediction model developed in this study showed errors ranging from $10.62 \%$ to $54.94 \%$. Hence, the regression model proved to be applicable to both construction systems: beamcolumn and structural masonry systems. Engineers and Architects can use it as a tool to estimate CDW in the Waste Management Plan of construction projects.
\end{abstract}

Keywords: $C D W$. Construction and demolition waste. Multiple linear regression model. Vertical residential buildings. Structural masonry system. 


\section{Introdução}

Entre as principais ferramentas legais relacionadas a gestão e gerenciamento de RCD no Brasil, estão a Lei $n^{0} 12.305$, que instituiu a Política Nacional de Resíduos Sólidos (BRASIL, 2010), e a Resolução $n^{\circ} 307$ do Conama (CONSELHO..., 2002). Ambas obrigam a elaboração, implantação e monitoramento de um PGRCD.

Como etapas integrantes do PGRCD, devem estar previstas: as estratégias de não geração, redução e minimização de resíduos; geração; classificação; segregação; acondicionamento; coleta; armazenamento temporário; transporte; tratamento e destinação final. A composição qualiquantitativa dos RCD é um dos processos de destaque, a partir do qual se estruturam as estratégias subsequentes do gerenciamento.

O relatório da Associação Brasileira de Empresas de Limpeza Pública e Resíduos Especiais (ASSOCIAÇÃO..., 2014) mostra que, no Brasil, foram coletados 122.262 ton/dia de RCD em 2014. Isso representa um indicador de 0,603 kg/hab.dia. A comparação com os dados do ano anterior permite uma estimativa do índice de incremento da geração desse tipo de resíduo no país, de cerca de 4,1\%. Embora o relatório limite-se apenas aos dados coletados nas organizações vinculadas à Abrelpe, percebe-se uma tendência cada vez maior da geração de RCD nos centros urbanos.

Índices de geração nessa magnitude também são apresentados por outros pesquisadores. Pode-se citar, por exemplo, indicadores iguais a: 0,55 kg/hab.dia para Passo Fundo, RS (BERNARDES et al., 2008); 0,33 kg/hab.dia para Fortaleza (OLIVEIRA et al., 2011); 0,998 kg/hab.ano em um município na região Noroeste do estado de São Paulo (ANGULO et al., 2011); 1,23 kg/hab.dia em Pelotas, RS (TESSARO; SÁ; SCREMIN, 2012).

Esse grande volume de resíduos significa necessidade urgente de implantação de ferramentas de gestão e gerenciamento para garantir a adequada proteção ambiental e saúde da população. Contudo, o correto gerenciamento de resíduos necessita, como fator primordial, um adequado levantamento qualiquantitativo.

Esse tema é relevante e discutido no mundo todo. Conforme Llatas (2011), Dias (2013), Ding e Xiao (2014), Sáez; Merino; Porras-Amores (2012) e Kern et al. (2015), conhecer a quantidade e a composição de RCD gerados é um desafio da sociedade atual e um fator preponderante para elaboração de projetos de gerenciamento de resíduos em canteiros de obras.
Em relação às dificuldades em caracterização dos RCD dois aspectos podem ser citados:

(a) a heterogeneidade na composição do RCD devido à variação das características locais e físicas de cada empreendimento e da respectiva variação com relação aos processos em que ocorre sua geração (TAVARES, 2007); e

(b) os processos de produção de obra ocorrem em instalações temporárias e cada obra é um produto diferente (DIAS, 2013).

Esses efeitos, no entanto, podem ser minimizados mediante planejamento e controle de obras. Um dos pontos de destaque, conforme diversos autores (SOUZA; DEANA, 2007; TAVARES, 2007; JOHN, 2000; KARPINSKI, 2009; MÁLIA; BRITO; BRAVO, 2011; DIAS, 2013; KERN et al., 2015), é que a geração de RCD é decorrente das perdas no processo construtivo. Segundo os mesmos autores, essas perdas começam a ocorrer na fase de projeto, na qual se tornam visíveis e onerosas e, posteriormente, na fase de construção.

Ainda que alguns resíduos não possam ser evitados, o potencial de redução de custos que há na prevenção de desperdícios na obra deve servir de incentivo a todos os intervenientes nas várias fases do ciclo de vida de uma obra. O objetivo é minimizar a produção de RCD (MÁLIA; BRITO; BRAVO, 2011).

Como exemplo de racionalização e combate à geração de resíduos nos canteiros de obra, Blumenschein (2007) cita o uso de blocos préfabricados em alvenarias (estruturais ou não) com estudo e projeto de modulação. Segundo o autor, este gera menor porcentagem de perdas do que em sistemas convencionais. $\mathrm{O}$ autor ainda conclui que a escolha adequada de tecnologias é fundamental ao processo construtivo para a redução de resíduos e perdas.

Tomando como exemplo as técnicas construtivas em alvenaria estrutural, Santos (1998) defende que a alvenaria estrutural, quando bem empregada, minimiza índices de desperdício. Assim, sua principal vantagem se dá no potencial de racionalização desse sistema.

Com relação à fase de concepção e projeto do empreendimento, ou produto, Souza e Deana (2007) afirmam que existe uma forte influência quanto à definição do futuro consumo de materiais por metro quadrado de obra a ser produzida. Conforme os autores, maiores ou menores compacidades afetam a quantidade de material requerido no projeto e na execução. 
Para Mascaró (2006), o grau de compacidade de um edifício pode ser representado matematicamente pelo índice econômico de compacidade (IeC), cujo cálculo se dá por meio da Equação 1.

$I e C=100 \times \frac{2 \times(A p \times \pi)^{0,5}}{P p r+(1,5 \times P c p)+\left(\frac{n A}{2}\right)}$

Onde:

Ap é a área superficial do projeto $\left(\mathrm{m}^{2}\right)$;

Ppr é o perímetro das paredes exteriores retas (m);

Pcp é o perímetro das paredes exteriores curvas (m); e

nA é o número e arestas das fachadas.

Assim, a importância da estimativa de geração de resíduos em obras está justificada por todos os aspectos anteriormente abordados. Apenas cabe ainda uma reflexão de que a redução de desperdícios e a utilização de melhores tecnologias na construção civil, além de todos os ganhos ambientais e sociais, também podem significar uma redução de custos da obra.

Todos esses fatores motivam pesquisas com predição de geração de RCD em canteiros de obras. Formoso et al. (2002) relatam que os primeiros estudos nacionais foram desenvolvidos por Pinto (1999) e Picchi (1993).

A pesquisa de Pinto (1999) considerou os dados disponibilizados pelos registros públicos e pesquisas com diversos agentes coletores em atuação, monitorados em um período de quatro anos. A conclusão é que a massa estimada para as edificações, executadas predominantemente por processos convencionais, é de $1.200 \mathrm{~kg} / \mathrm{m}^{2}$. A taxa de geração de RCD foi calculada em $150 \mathrm{~kg} / \mathrm{m}^{2}$, considerando a densidade, ou a massa unitária dos $\mathrm{RCD}$, de $1,2 \mathrm{t} / \mathrm{m}^{3}$.

Llatas (2011) também propôs um modelo que permitiu a estimativa de geração durante a fase de concepção da obra. Os resultados da aplicação do modelo proposto demonstraram que as maiores gerações de RCD estão relacionadas às etapas de pintura, limpeza do terreno e estrutura.

Já o método abordado por Sáez; Merino; PorrasAmores (2012) se utiliza de uma série de projetos de novos edifícios residenciais na Espanha. O modelo apresenta três indicadores para predição. Um indicador global de geração de RCD; um indicador que estima a geração de resíduos discriminando-a de acordo com a categoria estabelecida por normas vigentes (no caso da Espanha, o Catálogo Europeu de Resíduos (European Waste Catalougue - EWC); e um indicador que segregue a geração de RCD de acordo com as etapas da obra.

Conforme os autores, esses indicadores foram denominados $i_{1}, \quad i_{2}$ e $i_{3}$. Para todos os três indicadores a área construída considerada no cálculo diz respeito à média da área superficial dos projetos estudados. O volume de resíduos considerado foi uma média dos resíduos gerados nas obras. Porém, para o indicador $i_{1}$ considerou-se uma média global; para $i_{2}$, a média dos resíduos por sua tipificação normativa e, para $i_{3}$, a média dos resíduos de acordo com a etapa da obra em que foram gerados.

A obtenção desses indicadores se deu por meio de uma pesquisa realizada considerando cinco fases:

(a) identificação do prédio de construção tipicamente espanhola;

(b) caracterização dos projetos analisados;

(c) determinação das etapas de construção;

(d) cálculo dos resíduos gerados durante a construção dos edifícios estudados; e

(e) desenvolvimento de um modelo empírico para prever as quantidades de resíduos.

O modelo considerou os diferentes tipos de resíduos e as diferentes fases de construção em que são gerados (SÁEZ; MERINO; PORRASAMORES, 2012).

Por fim, cita-se o modelo proposto por Dias (2013), que fez uma análise estatística utilizando como ferramenta a regressão linear múltipla. O objetivo foi analisar a influência de variáveis de projeto arquitetônico e sistema produtivo sobre a geração de resíduos em obras residenciais verticais.

Os dados foram coletados em dezoito obras realizadas entre 2008 e 2013, na região metropolitana de Porto Alegre, RS. Na fase de tratamento dos dados, Dias (2013) considerou uma variável dependente e dois tipos de variáveis independentes, das quais a variável dependente está relacionada à quantidade de resíduo gerada. As variáveis independentes consideradas foram: as características relacionadas ao projeto arquitetônico e as características relacionadas à produção, sendo as primeiras variáveis quantitativas e as outras variáveis qualitativas.

As variáveis relacionadas ao projeto, segundo o método de Dias (2013), foram: área total construída $\left(\mathrm{m}^{2}\right)$, área do pavimento tipo $\left(\mathrm{m}^{2}\right)$, número de pavimentos, relação entre o número de pavimentos tipo e o número total de pavimentos do prédio (tipo/total), densidade de paredes internas no pavimento tipo $\left(\mathrm{m} / \mathrm{m}^{2}\right)$ e IeC. Já as variáveis 
relacionadas ao sistema de produção seriam compostas do: sistema produtivo (escala de 1 a 3 ), organização no canteiro ( 1 a 5 ) e reaproveitamento de resíduos no canteiro (sim ou não).

Aplicando-se o método de regressão linear múltipla utilizando o SPSS, o melhor modelo de predição determinado, conforme Dias (2013), está apresentando na Equação 2. $V R=-5202,886+\left(5138,519 \times \frac{T}{T}\right)+(1,411 \times A T P)+$
$(22,968 \times I e C)+(375,155 \times S P)+(-783,296 \times R R)+\epsilon$

Eq. 2

Onde:

VR total de resíduos;

T/T é o tipo/total (relação do número de pavimentos tipo pelo total de pavimentos do edifício);

ATP é a área do pavimento tipo do edifício a construir, em $\mathrm{m}^{2}$;

IeC é o índice econômico de compacidade;

SP é o sistema produtivo (escala de 1 a 3);

RR é o reaproveitamento de resíduos (0 (não pratica reaproveitamento) ou 1 (pratica o reaproveitamento); e

$\epsilon$ é o erro (variação estatística, característica do modelo de regressão linear múltipla).

Essa última pesquisa apresentada por Dias (2013) e a pesquisa publicada por Kern et al. (2015) limitaram-se ao desenvolvimento de um modelo de predição aplicável a obras verticais em concreto armado. O erro padrão do modelo foi de 301,1326 e $\mathrm{R}^{2}$ ajustado de 0,694 , ou seja, quase $70 \%$ da variação do volume de resíduos estão relacionados com as variáveis apresentadas na equação, o que, segundo Montgomery e Runger (2003), pode ser considerado como um bom resultado. Contribuindo para essas limitações, este artigo propôs uma modificação no modelo de regressão linear de Dias (2013). O objetivo foi o desenvolvimento de um modelo para predição de RCD em obras verticais aplicável para obras em alvenaria estrutural.

\section{Metodologia}

A metodologia é descrita em duas etapas:

(a) obtenção do banco de dados e elaboração de um modelo de regressão para estimativa de RCD em obras com alvenaria estrutural - ampliação do modelo de Dias (2013); e

(b) apresentação, aplicação e validação do modelo de regressão gerado em uma obra estudo de caso (obra Y).
Nesta pesquisa, por questões de confidencialidade, a empresa Estudo de Caso é denominada "empresa X”. Da mesma forma, a obra em andamento durante a pesquisa é denominada "obra Y". Assim, qualquer menção a dados de outras obras já concluídas se dá de maneira discreta, a fim de elucidar questões relativas ao escopo do trabalho sem revelar a identidade da empresa.

\section{Banco de dados para elaboração do modelo de regressão}

Utilizou-se uma ferramenta de pesquisa quantitativa e qualitativa, aplicando-se o método de estimativa de resíduos de Dias (2013) e Kern et al. (2015). O objetivo foi traçar um perfil do gerenciamento de RCD nos processos de construção dos edifícios residenciais verticais da empresa $X$, uma vez que a equação, elaborada estatisticamente nesse método, não só estima a quantidade de resíduos gerados, mas também aponta quais os principais fatores que influenciam na sua geração.

Nesse sentido, cabe abordar a definição de regressão linear múltipla, principal ferramenta estatística adotada pela metodologia de quantificação de resíduos de Dias (2013). Segundo Stevenson (1981), a regressão linear consiste na análise de dados amostrais, para uma determinada população, de forma a determinar a relação entre as variáveis depedentes e independentes. O produto dessa análise é uma equação que descreve o relacionamento entre as variáveis em termos matemáticos. A regressão linear múltipla evolve três ou mais variáveis. Há somente uma variável dependente (ou resposta), e as demais são independentes (STEVENSON, 1981).

Por se tratar de uma equação proveniente de um modelo estatístico de análise multivariada, é interessante ressaltar os parâmetros estatísticos que validaram essa equação, e que comprovam a influência das variáveis na geração de resíduos de construção civil. Os principais parâmetros, isto é, aqueles que se fazem necessários para a compreensão do modelo de estimativa adotado frente à aplicação no estudo de caso são: o teste de normalidade para uma amostra pequena (isto é, com tamanho menor do que 30, tais como os testes de Kolmogorov-Smirnov e de Shapiro-Wilk), o teste de correlação de Pearson (intrínseco ao método, utilizado para verificar o grau e o tipo de relacionamento das variáveis independentes com a dependente e das variáveis entre si), o coeficiente de determinação $\left(\mathrm{R}^{2}\right)$, o erro, e, finalmente, o nível de significância (HAIR JUNIOR et al., 2005; MONTGOMERY; RUNGER, 2003; STEVENSON, 1981). 
No caso do modelo de estimativa de Dias (2013), inicialmente foram coletados dados com base em vinte amostras, porém o modelo final utilizou apenas dezoito amostras. A autora retirou duas amostras durante o tratamento de dados, com o intuito de melhorar o ajustamento do modelo, pois elas demonstraram desvios elevados com relação ao volume de resíduos efetivo gerado pelas obras já concluídas.

Dessas duas amostras retiradas do banco de dados do modelo, uma era em alvenaria estrutural, amostra que apresentou maior diferença de estimativa de geração de RCD com relação ao volume efetivo gerado pela obra, com uma variação de 63,5\%, segundo Dias (2013). Com isso a autora definiu um modelo de regressão específico para tipo de construção viga-pilar, não sendo aplicável para alvenaria estrutural.

Assim, para a pesquisa proposta por este artigo, buscou-se a criação de um novo modelo que pudesse predizer o volume de resíduos de obras em alvenaria estrutural. O modelo foi estruturado a partir de observações de obras já concluídas e baseando-se no modelo estatístico desenvolvido por Dias (2013).

Como ferramenta eletrônica, utilizou-se o programa IBM SPSS Statistics versão 22. Todas as hipóteses foram testadas com nível de significância de $5 \%(p<0,05)$.

Iniciou-se com a tentativa de um modelo singular, o qual pudesse predizer o volume de RCD gerado nas obras em alvenaria estrutural da empresa X. Para tanto, procurou-se informações em empresa relativas às obras em alvenaria estrutural executadas nos últimos cinco anos, com enfoque nas variáveis iniciais do modelo de Dias (2013).

Porém, a empresa $\mathrm{X}$ disponibilizou dados confiáveis de apenas três obras nesse âmbito, denominadas afins de confidencialidade de obras $\mathrm{R}$, $\mathrm{S}$ e T. O volume de resíduos dessas obras foi obtido por meio do histórico emitido junto ao software de gestão utilizado pela empresa. As variáveis independentes quantitativas foram obtidas por meio da análise dos projetos arquitetônicos, e as variáveis qualitativas mediante informações coletadas em entrevistas com os engenheiros das obras.

Sobre as variáveis qualitativas apresentadas na Tabela 1 a seguir, estas foram obtidas por meio de relatos dos engenheiros responsáveis e registros fotográficos. Cabe ressaltar que, uma vez que as obras são da mesma empresa/construtora, com um período curto de tempo entre suas conclusões, as variáveis sistema produtivo e reaproveitamento de resíduos não variaram nesse caso. Esse fato contribui para a necessidade de se ampliar o número de amostras, a fim de se ter representatividade estatística para outros níveis de sistema produtivo e reaproveitamneto de resíduos.

Com isso, as variáveis estatísticas do modelo resultaram em um $\mathrm{R}^{2}$ igual a 1 , não sendo possível determinar o teste $\mathrm{F}$ e valor de significância. Isso é justificado pelo número de amostras que se mostrou demasiadamente pequeno para a técnica de regressão linear múltipla, conforme Hair Junior et al. (2005). Assim, o modelo singular não pôde ser considerado.

Dessa forma, foi criado então um banco de dados para essas três obras, concluídas entre 2011 e 2014, de acordo com as variáveis independentes iniciais consideradas no modelo de Dias (2013). A Tabela 1 apresenta esses dados.

A respeito das características das obras, a obra $\mathrm{R}$ era composta de quatro edifícios residenciais, em que três desses edifícios possuíam planta baixa idêntica e todos com cinco pavimentos. A obra S também era composta de quatro edifícios, sendo um diferente dos demais, com relação à área do pavimento tipo. Por fim, a obra T era composta de dois edifícios idênticos entre si e um diferente, com a área do pavimento tipo menor, todos com cinco pavimentos.

Assim, optou-se por ampliar o modelo de estimativa de Dias (2013) acrescentando ao banco de dados do modelo as obras $\mathrm{R}, \mathrm{S}$ e $\mathrm{T}$, no intuito de proporcionar maior representatividade ao modelo com relação às obras em alvenaria estrutural da empresa. Buscou-se viabilizar a sua utilização à empresa $\mathrm{X}$, e com um objetivo secundário de, no mínimo, manter a confiabilidade estatística do modelo.

Para esse caso também se utilizou o programa IBM SPSS Statistics versão 22 para obtenção das variáveis do modelo e para execução dos testes estatísticos necessários, os quais estão detalhados nos resultados. Da mesma forma, todas as hipóteses foram testadas com nível de significância de $5 \%(p<0,05)$.

Cabe ressaltar que as obras da empresa $\mathrm{X}$ pesquisadas possuem mais de um edifício para cada obra. Portanto, para a estimativa do volume de RCD gerado nessas obras, a exemplo do desenvolvimento do método de Dias (2013), realizou-se uma compilação a fim de homogeneizar a amostra, fazendo-se uma média das variáveis de projeto "IeC" e "área do pavimento tipo”. 


\section{Validação do modelo de regressão}

Com a determinação da ampliação do modelo de regressão de Dias (2013), fez-se uma aplicação deste em uma obra em andamento (obra Y da empresa X). Essa aplicação objetivou a validação do modelo.

\section{Caracterização do estudo de caso}

O objeto de estudo de caso, denominado no presente trabalho de "obra Y”, é um residencial localizado próximo ao centro comercial de Cachoeirinha (região metropolitana de Porto Alegre, Rio Grande do Sul (RS), composto de cinco edifícios em alvenaria estrutural e lajes préfabricadas de oito pavimentos e um prédio garagem de quatro pavimentos em concreto armado moldado in loco no sistema de lajes nervuradas com escoras metálicas, formas de pilares com pontalete e sarrafo metálicos (de modo que somente a chapa de compensado naval é de madeira).

Os apartamentos possuíam uma média de $50 \mathrm{~m}^{2}$, sendo o maior deles de 68,26 $\mathrm{m}^{2}$, na Torre 1 e o menor de $38,56 \mathrm{~m}^{2}$ no térreo das Torre 2 e 3 . As Torres 2 e 3 e as Torres 4 e 5 são iguais entre si, sendo os projetos arquitetônico, elétrico, hidrossanitário, estrutural, de fundações e demais projetos complementares compartilhados. A Torre 1 contava com um projeto singular com relação aos demais. As torres eram distribuídas perifericamente ao redor do terreno e prédio garagem no centro do terreno, comportando em seu subsolo apenas os reservatórios que alimentam as cinco torres residenciais, e, no térreo, além de vagas de estacionamento, parte da área de uso comum do condomínio. Cada pavimento desse prédio garagem possuía 1.697,7 m².

Nessas cinco torres são distribuídas 352 unidades habitacionais, sendo oito apartamentos por andar nas Torres 1, 4 e 5, e dez apartamentos por andar nas Torres 2 e 3 , com 362 vagas de estacionamento, a maior parte no prédio garagem, distribuído em um terreno de 10.811,49 m². A obra $\mathrm{Y}$ totaliza 28.039,03 $\mathrm{m}^{2}$ de área aconstruída e observou-se que o fluxo de produção da obra se deu no sentido anti-horário em planta, sendo que o edifício mais avançado em sua execução foi a Torre 3, seguida da Torre 1, Torre 2, edifício garagem, Torre 5 e Torre 4 . A Figura 1 mostra a planta de situação do empreendimento, cedida pela empresa X.

Tabela 1 - Banco de dados para inserção das obras concluídas amostradas

\begin{tabular}{|c|c|c|c|c|c|}
\hline \multirow{2}{*}{\multicolumn{3}{|c|}{ Amostra }} & \multicolumn{3}{|c|}{ Variável dependente } \\
\hline & & & \multicolumn{3}{|c|}{$\operatorname{VR}\left(\mathrm{m}^{2}\right)$} \\
\hline \multicolumn{3}{|c|}{$\mathrm{R}$} & \multicolumn{3}{|c|}{474} \\
\hline \multicolumn{3}{|c|}{$\mathrm{S}$} & \multicolumn{3}{|c|}{434} \\
\hline \multicolumn{3}{|c|}{$\mathrm{T}$} & \multicolumn{3}{|c|}{279} \\
\hline \multicolumn{6}{|c|}{ Variáveis quantitativas } \\
\hline $\mathrm{N}^{0}$ de Pav. & Tipo/Total & $\begin{array}{c}\text { Área total } \\
\left(\mathbf{m}^{2}\right)\end{array}$ & $\begin{array}{c}\text { Área Pav. Tipo } \\
\left(\mathrm{m}^{2}\right)\end{array}$ & IeC & Densidade das paredes \\
\hline 5 & 0,800 & 9705,540 & 460,670 & 44,940 & 0,810 \\
\hline 5 & 0,800 & 15250,890 & 756,670 & 37,240 & 0,810 \\
\hline 5 & 0,800 & 7738,060 & 481,340 & 44,120 & 0,800 \\
\hline
\end{tabular}

Variáveis qualitativas

\begin{tabular}{c|c|c}
\hline Organização do canteiro & Sistema produtivo & Reaproveitamento de resíduos \\
\hline 4 & 2 & 1 \\
\hline 3 & 2 & 1 \\
\hline 4 & 2 & 1 \\
\hline
\end{tabular}

Nota: Legenda:

VR (volume total de resíduos);

leC (Índice econômico de compacidade);

Organização do canteiro (1 - canteiros com falhas de organização - 5 para os canteiros mais organizados):

Sistema produtivo (1 - Artesanal; 2 - sistema intermediário quanto à industrialização; 3 - sistema construtivo

com práticas industrializadas); e

Reaproveitamento de resíduos (1 - Sim; 0 - Não).

314 Caetano, M. O.; Fagundes, A. B.; Gomes, L. P. 
Figura 1 - Representação da planta de situação da obra Y

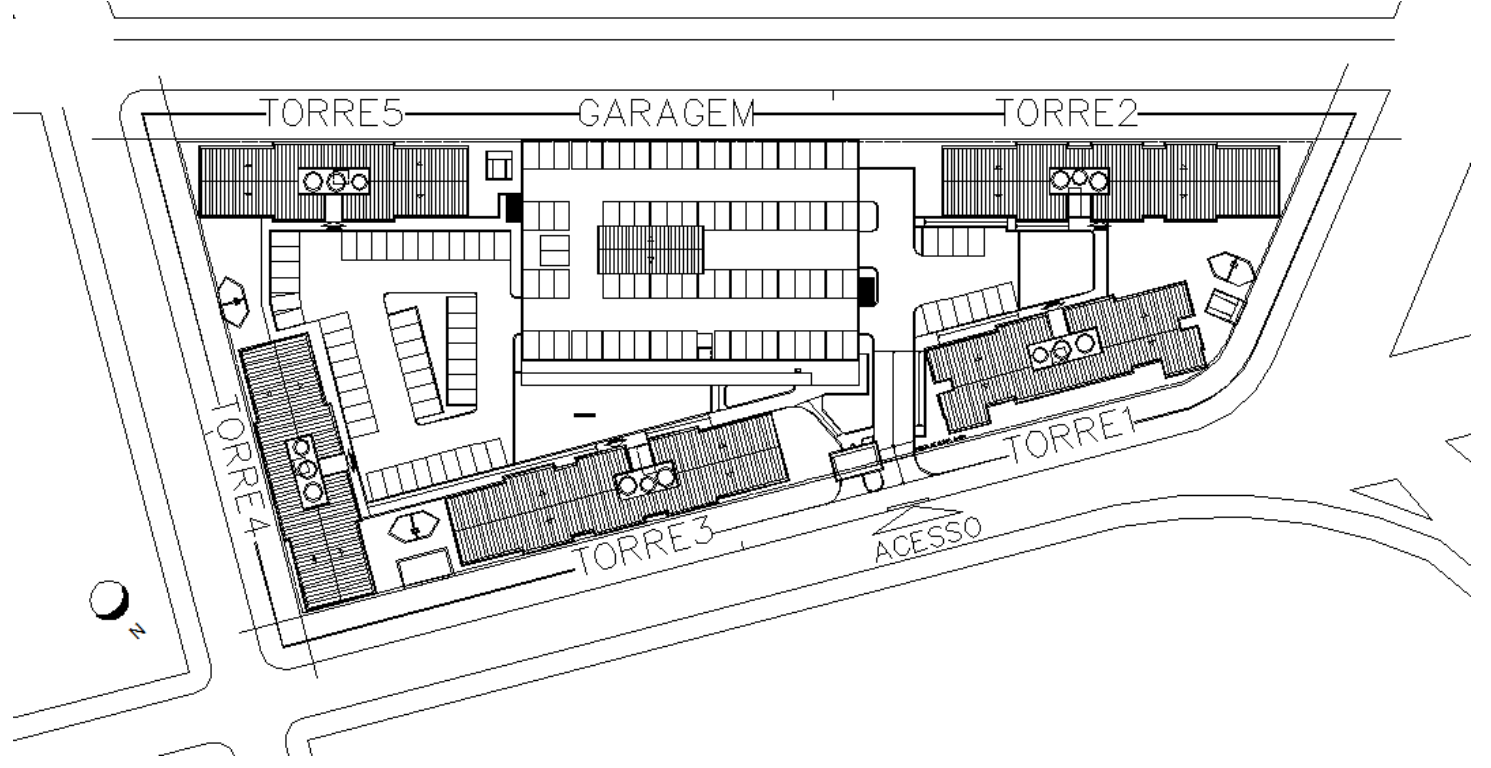

O planejamento da obra era dividido em três tipos de serviços civis: o de habitação, o de uso comum e o de infraestrutura. A fase em que a obra se encontrava no período de coleta de dados e aplicação do modelo de regressão abrangia somente os serviços de habitação, os quais foram estudados e dos quais foram selecionadas e padronizadas as etapas de serviço em andamento, ou já executadas, desde o início da obra Y (Tabela 2). Foram estas:

(a) infraestrutura;

(b) alvenaria estrutural de bloco cerâmico;

(c) tubulações elétricas e hidrossanitárias;

(d) instalações de gás;

(e) massa única interna;

(f) revestimento cerâmico;

(g) massa única externa; e

(h) supraestrutura edifício garagem.

Ainda para caracterização da obra Y, primeiramente fez-se uma avaliação qualiquantiativa dos resíduos de construção e demolição gerado pela obra. Este estudo está apresentado na seção "Resultados”.

\section{Aplicação do modelo de regressão na obra estudo de caso}

Com a elaboração do modelo de regressão proposto por esta pesquisa, fez-se a estimativa da geração de RCD nos edifícios em alvenaria estrutural da obra Y. Por fim, fez-se uma análise das variáveis independentes intrínsecas ao método e comparou-se a estimativa gerada pelo novo modelo à estimativa realizada no PGRCD da obra, o qual se baseava no indicador proposto por Pinto (1999).

\section{Resultados}

\section{Modelo de regressão para estimativa de RCD aplicável a obras com alvenaria estrutural}

Com o banco de dados das obras da empresa X, fez-se a aplicação direta do modelo de regressão de Dias (2013). O resultado entre a comparação da predição em relação ao volume de RCD medido em obra mostrou grande variação, em especial nas obras $\mathrm{S}$ e $\mathrm{T}$, ratificando a inadequação da utilização do modelo a obras no sistema de alvenaria estrutural. A Tabela 3 demonstra esses resultados.

Tal situação pode ser explicada pelo fato de que, no desenvolvimento do modelo de Dias, (2013), apenas uma obra, isto é, 5,55\% das amostras do modelo final, é em alvenaria estrutural. Essa única amostra apresentou maior diferença de estimativa de geração de RCD com relação ao volume gerado pela obra.

Assim, juntamente com essas amostras (R, S e T), inseriu-se também a amostra que havia sido excluída do modelo de Dias (2013), obra U, obtendo-se um total de 22 amostras. Esse novo número de amostras vem a atender o número mínimo sugerido por Hair Junior et al. (2005), que afirmam que análises com menos de 20 observações são apropriadas apenas à regressão simples. 
Tabela 2 - Identificação das etapas concluídas e em andamento até o período de coleta de dados na obra $Y$

\begin{tabular}{|c|c|c|}
\hline Identificação & Etapa construtiva & $\begin{array}{c}\text { Etapa concluída até a realização } \\
\text { do estudo de caso (\%) }\end{array}$ \\
\hline \multicolumn{3}{|c|}{ Torre 3} \\
\hline 1 & Infraestrutura & 100 \\
\hline 2 & Alvenaria estrutural de bloco cerâmico & 100 \\
\hline 3 & Tubulações elétricas e hidrossanitárias & 100 \\
\hline 4 & Instalações de gás & 88 \\
\hline 5 & Massa única interna & 100 \\
\hline 6 & Revestimento cerâmico & 63 \\
\hline 7 & Massa única externa & 100 \\
\hline \multicolumn{3}{|c|}{ Torre 1} \\
\hline 1 & Infraestrutura & 100 \\
\hline 2 & Alvenaria estrutural de bloco cerâmico & 100 \\
\hline 3 & Tubulações elétricas e hidrossanitárias & 100 \\
\hline 4 & Instalações de gás & 63 \\
\hline 5 & Massa única interna & 88 \\
\hline 6 & Revestimento cerâmico & 63 \\
\hline \multicolumn{3}{|c|}{ Torre 2} \\
\hline 1 & Infraestrutura & 100 \\
\hline 2 & Alvenaria estrutural de bloco cerâmico & 100 \\
\hline 3 & Tubulações elétricas e hidrossanitárias & 100 \\
\hline 4 & Instalações de gás & 63 \\
\hline 5 & Massa única interna & 88 \\
\hline 6 & Revestimento cerâmico & 63 \\
\hline \multicolumn{3}{|c|}{$\begin{array}{ll}\text { Edifício garagem } \\
\end{array}$} \\
\hline 1 & Infraestrutura & 100 \\
\hline 8 & Supraestrutura edifício garagem & 63 \\
\hline \multicolumn{3}{|c|}{ Torre 5} \\
\hline 1 & Infraestrutura & 100 \\
\hline 2 & Alvenaria estrutural de bloco cerâmico & 13 \\
\hline 3 & Tubulações elétricas e hidrossanitárias & 13 \\
\hline 5 & Massa única interna & 13 \\
\hline \multicolumn{3}{|c|}{ Torre 4} \\
\hline 1 & Infraestrutura & 100 \\
\hline 2 & Alvenaria estrutural de bloco cerâmico & 13 \\
\hline 3 & Tubulações elétricas e hidrossanitárias & 13 \\
\hline 5 & Massa única interna & 13 \\
\hline
\end{tabular}

Tabela 3 - Comparativo modelo de Dias (2013) em oposição ao montante Informado

\begin{tabular}{|c|c|c|c|}
\hline \multicolumn{4}{|c|}{ Geração de RCD $\left(\mathrm{m}^{3}\right)$} \\
\hline ID & $\begin{array}{l}\text { VR informado pela } \\
\text { empresa } X\left(\mathrm{~m}^{3}\right)\end{array}$ & $\begin{array}{c}\text { VR estimado }\left(\mathrm{m}^{3}\right) \text { (modelo } \\
\text { de Dias, } 2013)\end{array}$ & Diferença (\%) \\
\hline $\mathrm{R}$ & 474 & 557,130 & 17,54 \\
\hline $\mathrm{S}$ & 434 & 797,933 & 83,86 \\
\hline $\mathrm{T}$ & 279 & 567,462 & 103,39 \\
\hline
\end{tabular}

Aplicou-se então o mesmo tratamento às 22 amostras, porém considerando as variáveis independentes estabelecidas no modelo final adotado por Dias (2013) e que deu origem à equação desenvolvida no referido trabalho. Isso objetivou corroborar com o método de estimativa adotado, na tentativa de aumentar o poder de predição do modelo no sentido de abrangê-lo às obras em estudo, isto é, no sistema de alvenaria estrutural, gerando um novo modelo. 
O banco de dados contendo as 22 amostras é apresentado na Tabela 4, sendo a obra U em alvenaria estrutural, excluída do banco de dados de Dias (2013).

Assim, primeiramente foi realizado um teste de normalidade para a variável utilizando o SPSS, o qual se apresenta na Tabela 5. A estatística do teste de Kolmogorov-Smirnov de 0,142 aceita a hipótese de normalidade da variável dependente, pois é inferior ao valor exigido pelo teste para a significância de 0,05. O teste de Shapiro-Wilk confirma essa hipótese por meio do p-valor igual a 0,085. Já o gráfico de caixa, ou Boxplot, na Figura 2 demonstra que não existem outliers no conjunto amostral da variável dependente.
Após esse procedimento, verificou-se a possibilidade de as variáveis do modelo explicarem o mesmo fenômeno, o que vem a ser chamado de multicolinearidade. Apesar de o estudo de Dias (2013) ter abordado esse aspecto com as variáveis dependentes consideradas, como foram adicionadas novas observações, repetiu-se então o procedimento, demonstrado na Tabela 6.

Constata-se que, de uma maneira geral, há um bom relacionamento entre as variáveis, porém sem que exista a possibilidade de grandes interferências entre elas a ponto de reduzir a parcimônia do modelo. Assim, obteve-se o limite de correlação positiva de 0,55 para "Reaproveitamento de resíduos” versus “Tipo/Total” e negativa de $-0,44$ para “Área do pavimento tipo” versus IeC.

Tabela 4 - Banco de dados do modelo de Dias (2013) adicionadas as amostras R, S, T e U

\begin{tabular}{c|c|c|c|c|c|c}
\hline Amostra & VR & Tipo/Total & $\begin{array}{c}\text { Área do pavimento tipo } \\
\mathbf{( m}^{2} \mathbf{)}\end{array}$ & IeC & $\begin{array}{c}\text { Sistema } \\
\text { construtivo }\end{array}$ & $\begin{array}{c}\text { Reaproveitamento } \\
\text { de resíduos }\end{array}$ \\
\hline B1* & 1410,00 & 0,81 & 321,9 & 62,83 & 2 & 0 \\
B2* & 1890,00 & 0,83 & 509,23 & 43,12 & 2 & 0 \\
B3* & 840,00 & 0,83 & 369,63 & 44,23 & 2 & 1 \\
B4* & 1074,00 & 0,85 & 429,04 & 56,31 & 2 & 1 \\
B5* & 1074,00 & 0,75 & 360,65 & 62,09 & 1 & 0 \\
B6* & 872,00 & 0,71 & 564,95 & 59,23 & 1 & 0 \\
B7* & 420,00 & 0,69 & 423,00 & 55,47 & 1 & 0 \\
B** & 612,00 & 0,82 & 346,8 & 60,58 & 1 & 1 \\
B9* & 736,00 & 0,81 & 399,77 & 66,82 & 1 & 1 \\
B10* & 1952,00 & 0,82 & 605,84 & 51,77 & 3 & 1 \\
B11* & 1188,00 & 0,77 & 532,00 & 60,17 & 3 & 1 \\
B12* & 1512,00 & 0,88 & 531,48 & 58,31 & 3 & 1 \\
B13* & 2164,00 & 0,93 & 696,57 & 55,69 & 3 & 1 \\
B14* & 876,00 & 0,78 & 546,83 & 51,93 & 3 & 1 \\
B15* & 1012,00 & 0,82 & 230,51 & 57,91 & 1 & 0 \\
B16* & 712,00 & 0,92 & 506,00 & 41,05 & 2 & 1 \\
B17* & 1165,00 & 0,75 & 419,2 & 61,74 & 2 & 0 \\
B18* & 2252,00 & 0,81 & 427,21 & 72,27 & 2 & 2 \\
R** & 474,00 & 0,80 & 460,67 & 44,94 & 2 & 1 \\
S** & 434,00 & 0,80 & 756,67 & 37,24 & 2 & 1 \\
T** & 279,00 & 0,80 & 481,34 & 44,12 & 2 & 1 \\
U** & 400,00 & 0,71 & 282,33 & 59,35 & 2 & 0 \\
\hline Not & & & &
\end{tabular}

Nota: Legenda:

VR (Volume total de resíduos);

leC (Índice econômico de compacidade);

Organização do canteiro (1 - canteiros com falhas de organização - 5 para os canteiros mais organizados);

Sistema produtivo ( 1 - Artesanal; 2 - sistema intermediário quanto à industrialização; 3 - sistema construtivo

com práticas industrializadas); e

Reaproveitamento de resíduos (1 - Sim; 0 - Não).

* Amostras do banco de dados de Dias (2013).

**Amostras do banco de dados do presente estudo.

Tabela 5 - Teste de normalidade para a variável dependente (VR)

\begin{tabular}{c|c|c|c|c|c|c}
\hline & \multicolumn{2}{|c|}{ Kolmogorov-Smirnov } & \multicolumn{3}{c}{ Shapiro-Wilk } \\
\hline \multirow{2}{*}{ VR } & Estatística & df & p-valor & Estatística & df & p-valor \\
\cline { 2 - 7 } & 0,142 & 22 & 0,2 & 0,992 & 22 & 0,085 \\
\hline
\end{tabular}


Figura 2 - Gráfico de caixa (boxplot) da variável dependente

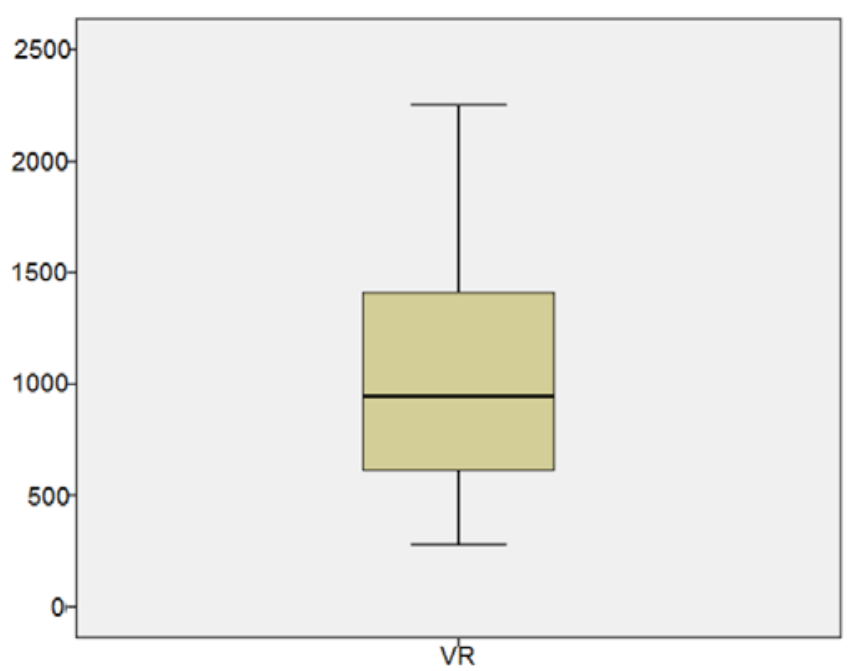

VR= Volume de Resíduos $\left(m^{3}\right)$

$\mathrm{N}=22$

Tabela 6 - Correlação de Pearson para as variáveis do modelo ampliado

\begin{tabular}{|c|c|c|c|c|c|c|}
\hline $\begin{array}{l}\text { Correlação de } \\
\text { Pearson }\end{array}$ & VR & Tipo/Total & $\begin{array}{c}\text { Área do } \\
\text { pavimento } \\
\text { tipo }\left(\mathrm{m}^{2}\right)\end{array}$ & IeC & $\begin{array}{l}\text { Reaproveitamento } \\
\text { de resíduos }\end{array}$ & $\begin{array}{c}\text { Sistema } \\
\text { construtivo }\end{array}$ \\
\hline VR & 1,00 & 0,42 & 0,23 & 0,33 & $-0,15$ & 0,45 \\
\hline Tipo/Total & 0,42 & 1,00 & 0,28 & $\begin{array}{c}- \\
0,26\end{array}$ & 0,55 & 0,42 \\
\hline $\begin{array}{l}\text { Área do pavimento } \\
\text { Tipo }\left(\mathrm{m}^{2}\right)\end{array}$ & 0,23 & 0,28 & 1,00 & 0,44 & 0,47 & 0,54 \\
\hline $\mathrm{IeC}$ & 0,33 & $-0,26$ & $-0,44$ & 1,00 & $-0,42$ & $-0,21$ \\
\hline $\begin{array}{l}\text { Reaproveitamento } \\
\text { de resíduos }\end{array}$ & - & 0,55 & 0,47 & $\overline{-}$ & 1,00 & 0,47 \\
\hline Sistema construtivo & 0,45 & 0,42 & 0,54 & $\begin{array}{c}- \\
0,21\end{array}$ & 0,47 & 1,00 \\
\hline
\end{tabular}

Finalmente, a Tabela 7 apresenta os resultados dos testes relativos à confiabilidade e variabilidade do modelo de regressão elaborado, e na Tabela 8 são demonstradas as constantes relativas a cada variável com os respectivos erros e valores de significância.

Constata-se então que todas as variáveis se mostram significativas ao nível de confiança de 95\%. Quanto ao nível de relacionamento da variável dependente com as variáveis independentes, o modelo apresenta o valor de $\mathrm{R}^{2}$ ajustado de 0,741 , sendo considerado um relacionamento positivo moderado. Em outras palavras, o modelo explica cerca de $74 \%$ da geração de resíduos em obras de edificações residenciais verticais, incluindo obras em alvenaria estrutural.
Outro dado importante intrínseco ao modelo é o teste $\mathrm{F}$, que comprovou que o modelo tem significância sobre a geração de resíduos, obtendo p-valor muito baixo. Assim, aceita-se a hipótese alternativa para a significância do modelo, evidenciando sua possibilidade de predição.

Logo, torna-se aceitável do ponto de vista estatístico e do ponto de vista conceitual, tendo em vista as análises realizadas neste estudo e as análises anteriores deste estudo desenvolvidas no modelo de estimativa proposto por Dias (2013). Com isso, o modelo de estimativa de RCD proposto por este artigo está apresentado na Equação 3.

$V R=-6581,931+\left(6286,927 \times \frac{T}{T}\right)+(1,616 \times A T P)+$ $(30,189 \times$ IeC $)+(331,328 \times S P)+(-787,682 \times R R)+\epsilon$

Eq. 3

Onde: 
VR é o volume total de resíduos;

T/T é o tipo/total (relação do número de pavimentos tipo pelo total de pavimentos do edifício);

ATP é a área do pavimento tipo do edifício a construir, em metros quadrados;

IeC é o índice econômico de compacidade;

SP é o sistema Produtivo (escala de 1 a 3);

RR é o reaproveitamento de resíduos (0 - não pratica reaproveitamento ou 1 - pratica o reaproveitamento); e

$\varepsilon$ é o erro (variação estatística, característica do modelo de regressão linear múltipla).

A fim de ratificar a adequabilidade do modelo, buscou-se comparar com o volume efetivo das obras da empresa $X$ (obras $R, S$ e $T$ ), com o método de Dias (2013) e com o modelo ampliado pelo presente trabalho, apresentando a diferença em percentual com relação ao volume gerado. A Tabela 9 demonstra esse comparativo.

Constata-se que o novo modelo foi capaz de predizer o volume de RCD (em metros cúbicos) com menor diferença entre o volume real de resíduos produzidos na obra com relação ao método de estimativa de Dias (2013). A diferença entre o volume informado pela empresa $\mathrm{X}$ com relação às obras $\mathrm{R}, \mathrm{S}$ e $\mathrm{T}$ chega a, no máximo, cerca de 54\% para o modelo apliado.

Já a comparação com o modelo de Pinto (1999), para esse mesmo caso, mostrou erros variando de 155,95\% a 339,25\%. A magnitude desses erros revelam que o modelo de Pinto (1999) precisa ser reavaliado. Os índices estimados pelo autor, e ainda utilizado amplamente nos PGRCDs, podem não representar a realidade das obras do país atualmente.

\section{Validação do modelo de regressão \\ Características dos RCD da obra Y}

As características dos RCD da obra estudo de caso foram determinadas utilizando os seguintes dados: PGRCD elaborado por uma empresa especializada contratada; ordens de serviço de transporte de resíduos acumuladas desde o início da obra junto ao agente almoxarife (emitidas pela empresa contratada responsável pelo transporte dos RCD); análise de contratos emitida por meio do software de gestão utilizado pela empresa $X$, junto ao setor de engenharia da obra Y (contendo o histórico de pagamentos efetuados à empresa responsável pelo transporte de RCD).

Tabela 7 - Resumo dos resultados da análise de regressão do modelo ampliado

\begin{tabular}{c|c|c|c|c}
\hline $\mathbf{R}^{2}$ & $\mathbf{R}^{2}$ ajustado & Erro padrão da estimativa & $\mathbf{F}$ & p-valor \\
\hline 0,802 & 0,741 & 298,464 & 13,001 & 0,00004 \\
\hline
\end{tabular}

Tabela 8 - Coeficientes do modelo ampliado

\begin{tabular}{l|c|c|c}
\hline \multicolumn{1}{c|}{ Coeficientes do modelo } & $\boldsymbol{\beta}$ & Erro padrão & p-valor \\
\hline (Constante) & $-6581,931$ & 1195,449 & 0,00005 \\
Tipo/Total & 6286,927 & 1321,742 & 0,00021 \\
Área do pavimento tipo $\left(\mathrm{m}^{2}\right)$ & 1,616 & 0,675 & 0,02924 \\
IEC & 30,189 & 8,418 & 0,00247 \\
Sistema construtivo & 331,328 & 116,018 & 0,01144 \\
Reaproveitamento de resíduos & $-787,682$ & 176,281 & 0,00039 \\
\hline
\end{tabular}

Tabela 9 - Comparativo do volume de RCD gerado versus o volume estimado no modelo de Dias (2013) ampliado e o volume estimado no modelo original de Dias (2013)

\begin{tabular}{|c|c|c|c|c|c|}
\hline \multirow[b]{2}{*}{ Obra } & \multirow{2}{*}{$\begin{array}{c}\text { VR informado } \\
\text { pela empresa } \\
X\left(\mathbf{m}^{3}\right)\end{array}$} & \multicolumn{2}{|c|}{ Predição pelo modelo ampliado } & \multicolumn{2}{|c|}{$\begin{array}{c}\text { Predição pelo modelo original de } \\
\text { Dias (2013) }\end{array}$} \\
\hline & & $\begin{array}{l}\text { VR - Geração de } \\
\text { RCD }\left(\mathbf{m}^{3}\right)\end{array}$ & $\begin{array}{c}\text { Diferença em relação } \\
\text { ao informado }(\%)\end{array}$ & $\begin{array}{l}\text { VR - Geração } \\
\text { de RCD }\left(\mathbf{m}^{3}\right)\end{array}$ & $\begin{array}{c}\text { Diferença em } \\
\text { relação ao } \\
\text { informado (\%) }\end{array}$ \\
\hline $\mathrm{R}$ & 474 & 423,646 & $-10,62$ & 557,13049 & 17,54 \\
\hline $\mathrm{S}$ & 434 & 669,461 & 54,25 & 797,93289 & 83,86 \\
\hline $\mathrm{T}$ & 279 & 432,289 & 54,94 & 567,4621 & 103,39 \\
\hline
\end{tabular}


Levantou-se, como parte da coleta quantitativa de dados, os projetos arquitetônicos, memoriais descritivos da obra e os cronogramas mensais e semanais, contendo as etapas de construção executadas até o período em estudo, bem como seus percentuais de conclusão.

A Tabela 10 apresenta os indicadores de geração de RCD da obra $\mathrm{Y}$, considerando o período de treze meses. A estimativa de geração de RCD foi realizada considerando o método de Sáez; Merino; Porras-Amores (2012). Porém, em vez de ser considerada uma área total construída, considerouse para cada etapa a área superficial construída de acordo com os cronogramas fornecidos pela equipe de engenharia da obra, uma vez que o método referenciado se dá com base em quantitativos de obras já concluídas.

Esses indicadores permitiram diagnosticar o volume de RCD gerado por etapa e por área superficial construída para a obra Y. Considerouse na Etapa 1 (Infraestrutura), o montante de RCD e a área superficial construída, também do edifício garagem, tendo em vista a homogeneização do indicador.

Os indicadores por etapa construtiva demonstram que, para os processos construtivos empregados na obra Y, as etapas que mais produzem resíduos são: massa única interna, alvenaria estrutural de bloco cerâmico e supraestrutura do edifício garagem.

Ainda, tendo como referência o método de Sáez; Merino; Porras-Amores (2012). considerou-se um indicador global de geração de RCD, apresentado na Tabela 11. Este, sim, considerou a área total construída, a fim de se diagnosticar a geração de resíduos da obra Y.

Os índices apresentados nas Tabelas 10 e 11, se comparados a outras pesquisas na área, retratam uma grande diferença de geração de RCD. Cita-se aqui, por exemplo, os indicadores relatados por: Llatas (2011) (estimativa de geração de 0,4351 $\mathrm{m}^{3} / \mathrm{m}^{2}$ de RCD das fases de acabamento); Sáez; Merino; Porras-Amores (2012) (taxa média de geração de RCD considerando a construção com placas de gesso igual a $0,188 \mathrm{~m}^{3} / \mathrm{m}^{2}$ e para alvenaria de tijolo convencional igual a 0,192 $\mathrm{m}^{3} / \mathrm{m}^{2}$ ); Ding e Xiao (2014) (geração de RCD, na Espanha, em 2009, para novas construções igual a $0,3076 \mathrm{~m}^{3} / \mathrm{m}^{2}$ e para demolições igual a 1,2676 $\mathrm{m}^{3} / \mathrm{m}^{2}$ ) e Kern et al. (2015) (geração de RCD variando de $0,05 \mathrm{~m}^{3} / \mathrm{m}^{2}$ até $0,37 \mathrm{~m}^{3} / \mathrm{m}^{2}$ de área total construída, para obras localizadas na região metropolitana de Porto Alegre, RS).

Essas diferenças quantitativas são justificadas por:

(a) tipo construtivo, já que a obra desta pesquisa refere-se à construção em alvenaria estrutural; e

(b) a obra em estudo não estava finalizada conforme pode ser detectado na Tabela 2.

Percebe-se, no entanto, algumas semelhanças com o trabalho de Caetano, Selbach e Gomes (2016). Na pesquisa, o indicador de geração de RCD para a etapa de acabamentos em edificações horizontais em alvenaria estrutural foi de $0,012 \mathrm{~m}^{3} / \mathrm{m}^{2}$.

Tabela 10 - Indicador de geração de RCD por etapa construtiva

\begin{tabular}{c|l|c|c|c}
\hline \multicolumn{4}{c}{ PERÍODO: treze meses de obra } \\
\hline $\begin{array}{c}\text { Etapa de } \\
\text { construção }\end{array}$ & \multicolumn{1}{c}{ Descrição } & Volume $\left.\mathbf{( m}^{3}\right)$ & $\begin{array}{c}\text { Superfície } \\
\text { construída }\left(\mathbf{m}^{\mathbf{2}}\right)\end{array}$ & $\mathbf{i}\left(\mathbf{( m}^{\mathbf{3}} / \mathbf{m}^{\mathbf{2}}\right)$ \\
\hline 1 & Infraestrutura & 56,89 & $28.039,03$ & $2,03 \times 10^{-3}$ \\
2 & Alvenaria estrutural de bloco cerâmico & 149,45 & $15.162,66$ & $9,86 \times 10^{-3}$ \\
3 & Tubulações elétricas e hidrossanitárias & 4,64 & $15.162,66$ & $3,06 \times 10^{-4}$ \\
4 & Instalações de gás & 1,47 & $10.120,34$ & $1,45 \times 10^{-4}$ \\
5 & Massa única interna & 159,31 & $14.017,61$ & $1,14 \times 10^{-2}$ \\
6 & Revestimento cerâmico & 4,26 & $8.864,60$ & $4,80 \times 10^{-4}$ \\
7 & Massa única externa & 8,00 & $5.022,96$ & $1,59 \times 10^{-3}$ \\
8 & Supraestrutura edifício garagem & 20,00 & $3.763,29$ & $5,31 \times 10^{-3}$ \\
\hline
\end{tabular}

Nota: Legenda: i (Geração de RCD por área superficial).

Tabela 11 - Indicador global de geração de RCD

\begin{tabular}{c|c|c}
\hline \multicolumn{3}{c}{ PERÍODO: treze meses de obra } \\
\hline Volume $\left(\mathbf{m}^{3}\right)$ & Superfície construída $\left(\mathbf{m}^{\mathbf{2}}\right)$ & $\mathbf{i}\left(\mathbf{m}^{\mathbf{3}} / \mathbf{m}^{\mathbf{2}}\right)$ \\
\hline 404,00 & $18.952,95$ & $2,13 \times 10^{-2}$ \\
\hline
\end{tabular}

Nota: Legenda: i (Geração de RCD por área superficial). 
Além do indicador "geração de resíduos por etapa construtiva" e do "indicador global”, determinouse 0 indicador qualitativo de composição gravimétrica dos RCD gerados na obra. Constatouse $54,55 \%$ de materiais enquadrados na Classe A e $45,45 \%$ na Classe B (Tabela 12), indicador que se difere, em absoluto, de obras típicas brasileiras conforme mostra a comparação realizada na Tabela 12. Certamente as diferenças estão relacionadas ao término da obra e ao tipo construtivo.

\section{Aplicação do modelo de regressão na obra estudo de caso}

Conforme apresentado anteriormente, constatada a adequabilidade e representatividade com relação às obras em alvenaria estrutural mantida a confiabilidade estatística do modelo, e, ainda, constatada uma menor diferença na estimativa de RCD para as obras em alvenaria estrutural já concluídas da empresa $\mathrm{X}$, aplicou-se o modelo ampliado de Dias (2013) à obra Y. Foram aplicadas as variáveis constatadas com as informações de projeto e da obra às respectivas constantes. Exemplifica-se o resultado na Tabela 13.

Tabela 12 - Comparativo da composição gravimétrica de RCD da obra Y com algumas composições abordadas pela bibliografia

\begin{tabular}{|c|c|c|c|c|c|c|c|c|}
\hline \multirow[b]{2}{*}{$\begin{array}{c}\text { Conama } \\
307(2002)\end{array}$} & \multirow[b]{2}{*}{ Componente } & \multicolumn{7}{|c|}{ Composição de RCD (\%) } \\
\hline & & \multicolumn{2}{|c|}{ Obra "Y" } & $\begin{array}{c}\text { Daltro } \\
\text { Filho et } \\
\text { al. } \\
(2006) \\
\end{array}$ & $\begin{array}{l}\text { Tessaro, } \\
\text { Sá e } \\
\text { Scremin } \\
(2012) \\
\end{array}$ & $\begin{array}{c}\text { Bernardes } \\
\text { et al. } \\
\text { (2008) }\end{array}$ & $\begin{array}{c}\text { Araújo e } \\
\text { Carnaúba } \\
(2010)\end{array}$ & $\begin{array}{l}\text { Ângulo } \\
\text { et al. } \\
\text { (2011) }\end{array}$ \\
\hline \multirow{3}{*}{$\begin{array}{l}\text { Classe A } \\
\text { (reutilizáveis ou } \\
\text { recicláveis como } \\
\text { agregados) }\end{array}$} & Argamassa & 22,73 & \multirow{3}{*}{54,55} & \multirow{3}{*}{63,55} & \multirow{3}{*}{88,00} & \multirow{3}{*}{$94,8 \%$} & \multirow{3}{*}{96,44} & \multirow{3}{*}{91} \\
\hline & Concreto & 18,18 & & & & & & \\
\hline & Cerâmicos & 13,64 & & & & & & \\
\hline \multirow{5}{*}{$\begin{array}{l}\text { Classe B } \\
\text { (recicláveis para } \\
\text { outras } \\
\text { destinações) }\end{array}$} & Papelão & \multirow{4}{*}{36,36} & \multirow{5}{*}{45,45} & \multirow{5}{*}{5,08} & \multirow{5}{*}{11,00} & \multirow{5}{*}{$3,1 \%$} & \multirow{5}{*}{1,31} & \multirow{5}{*}{9} \\
\hline & Papel & & & & & & & \\
\hline & Metal & & & & & & & \\
\hline & $\begin{array}{l}\text { Plástico } \\
\text { Madeira }\end{array}$ & & & & & & & \\
\hline & Gesso & - & & & & & & \\
\hline $\begin{array}{l}\text { Classe C } \\
\text { (resíduos para } \\
\text { os quais não } \\
\text { foram } \\
\text { desenvolvidas } \\
\text { tecnologias ou } \\
\text { aplicações } \\
\text { economicamente } \\
\text { viáveis) }\end{array}$ & - & - & - & 29,25 & - & $2,1 \%$ & - & - \\
\hline $\begin{array}{l}\text { Classe D } \\
\text { (perigosos) }\end{array}$ & - & - & - & 2,12 & - & - & 0,46 & - \\
\hline
\end{tabular}

Tabela 13 - Estimativa de geração de resíduos na obra Y (edifícios em alvenaria estrutural), utilizando o modelo ampliado (Torres 1 a 5)

\begin{tabular}{l|c|c|c}
\hline \multicolumn{1}{c|}{ Coeficientes do modelo } & $\boldsymbol{\beta}$ & $\mathbf{X}$ & $\boldsymbol{\beta . X}$ \\
\hline (Constante) & $-6581,93$ & 1 & $-6581,931$ \\
Tipo/Total & 6286,927 & 0,875 & 5501,061 \\
Área do pavimento tipo $\left(\mathrm{m}^{2}\right)$ & 1,615792 & 550,440 & 889,397 \\
IEC & 30,18947 & 58,912 & 1778,524 \\
Sistema construtivo & 331,3276 & 2 & 662,655 \\
Reaproveitamento de resíduos & $-787,682$ & 1 & $-787,682$ \\
\hline \multicolumn{2}{r|}{} \\
\hline
\end{tabular}


Pode-se perceber que as variáveis “Tipo/Total”, "Sistema produtivo" e "Reaproveitamento de resíduos" se tornam constantes, uma vez que se trata da mesma obra, mantendo as características de influência dessas variáveis constantes para o caso específico da obra Y.

Comparou-se então o montante de RCD estimado na obra Y por meio do método gerado no trabalho versus o montante de RCD estimado pelo PGRCD (PINTO, 1999) e o modelo original de Dias (2013). A análise da Tabela 14 demonstra uma diferença significativa quando aplicado o indicador proposto por Pinto (1999). Isso reforça a necessidade de rever os índices utilizados nos PGRCDs das obras no país. Os resultados desta pesquisa realçam as diferenças elevadas entre o que realmente é gerado nas obras e a estimativa proposta por Pinto (1999).

Percebe-se, no entanto, uma similaridade entre o modelo original de Dias (2013) e o modelo desenvolvido por esta pesquisa. Isso fica mais evidente na predição considerando as torres em alvenaria estrutural e o edifício garagem em concreto armado, demonstrando assim a aplicação do modelo modificado para ambos os tipos construtivos.

A semelhança da predição considerando o modelo de Dias (2013) e o modelo ampliado é justificada pelo método de estimativa utilizado (regressão múltipla). Assim, a análise dos erros obtidos nas Tabelas 9 e 14 mostra boa adequabilidade do modelo ampliado. Isso permite considerar que o modelo desenvolvido pode ser considerado na estimava de geração de RCD tanto para o tipo construtivo viga-pilar quanto para alvenaria estrutural, e até mesmo um tipo construtivo misto considerando essas duas tecnologias.

Por fim, cabe ainda uma ressalva de que o fato de algumas etapas da obra "Y" não estarem 100\% finalizadas (ver Tabela 2) não promoveu influência na validação do modelo de predição proposto, o que é explicado pelas variáveis. Nenhuma delas envolve diretamente a etapa construtiva. Outro ponto é que a única que relaciona a caracterização de RCD refere-se a "Reaproveitamento de resíduos”, uma variável binária (0 - não pratica reaproveitamento ou 1 - pratica o reaproveitamento), ou seja, independente da etapa da obra.

\section{Conclusões}

O modelo de regressão múltipla para estimativa da geração de resíduos de obra em empreendimentos residenciais verticais em alvenaria estrutural, desenvolvido por esta pesquisa, mostrou-se aplicável para o tipo construtivo viga-pilar, para alvenaria estrutural e para um tipo construtivo misto considerando essas duas tecnologias. É um modelo que permite a generalização para os tipos construtivos viga-pilar e/ou para alvenaria estrutural, podendo ser utilizado nos PGRCDs, em substituição ao modelo de Pinto (1999).

Considerando um intervalo de confiança de 95\%, o modelo de predição apresentou um coeficiente de determinação $\left(\mathrm{R}^{2}\right)$ de 0,802 e coeficiente de determinação ajustado ( $\mathrm{R}^{2}$ ajustado) de 0,741 , ou seja, explica cerca de $74 \%$ da geração de resíduos em obras de edificações residenciais verticais, incluindo obras em alvenaria estrutural.

A comparação da quantidade real de RCD gerada na obra informada pela empresa (VR), com a estimativa gerada pelo modelo de predição desenvolvido por esta pesquisa, mostrou erros na faixa de $-10,62 \%$ até $54,94 \%$, ou seja, para as tecnologias construtivas avaliadas, o modelo se mostrou mais preciso do que o proposto por Pinto (1999) e Dias (2013). Estes apresentaram erros variando entre $155,95 \%$ - 339,25\% e $17,54 \%$ 83,86\%, respectivamente.

Tabela 14 - Comparativo do método adotado pelo PGRCD da obra Y versus o modelo de estimativa ampliado

\begin{tabular}{|c|c|c|c|c|c|}
\hline \multirow[b]{2}{*}{ Obra Y } & \multirow{2}{*}{$\begin{array}{c}\text { Geração de } \\
\text { RCD segundo } \\
\text { modelo } \\
\text { ampliado }\left(\mathbf{m}^{3}\right)\end{array}$} & \multicolumn{2}{|c|}{$\begin{array}{c}\text { Predição pelo método de Pinto } \\
\text { (1999) }\end{array}$} & \multicolumn{2}{|c|}{$\begin{array}{c}\text { Predição pelo modelo original } \\
\text { de Dias (2013) }\end{array}$} \\
\hline & & $\begin{array}{c}\text { VR - } \\
\text { Geração de } \\
\operatorname{RCD}\left(\mathbf{m}^{3}\right) \\
\end{array}$ & $\begin{array}{c}\text { Diferença em } \\
\text { relação modelo } \\
\text { ampliado (\%) }\end{array}$ & $\begin{array}{c}\text { VR - } \\
\text { Geração de } \\
\operatorname{RCD}\left(\mathbf{m}^{3}\right) \\
\end{array}$ & $\begin{array}{l}\text { Diferença em } \\
\text { relação modelo } \\
\text { ampliado (\%) }\end{array}$ \\
\hline Torres 1- 5 & 1462,024 & 2752,22 & 88,25 & 1390,095 & $-4,92$ \\
\hline $\begin{array}{l}\text { Torres } 1-5 \text { e } \\
\text { edifício garagem }\end{array}$ & 1968,58 & 3504,92 & 78,04 & 1815,16 & $-7,79$ \\
\hline
\end{tabular}

322 Caetano, M. O.; Fagundes, A. B.; Gomes, L. P. 
Em relação à validação do modelo e aplicação na obra estudo de caso, percebeu-se, comparando o modelo desenvolvido em relação à estimativa de Pinto (1999), uma diferença de estimativa na faixa de $78,04 \%$ até $88,25 \%$. Essa mesma comparação com o modelo de Dias (2013) mostrou erros na faixa de $-4,92 \%$ até $-7,79 \%$. Essa semelhança da predição é justificada pelo uso do mesmo método de estimativa, ou seja, regressão múltipla, e do mesmo banco de dados, porém ampliado.

\section{Referências}

ANGULO, S. C. et al. Resíduos de Construção e Demolição: avaliação de métodos de quantificação. Engenharia Sanitária e Ambiental, v. 16, n. 3, p. 299-306, jul./set. 2011.

ARAÚJO, N. M. C.; CARNAÚBA, T. M. G. V. Composição Gravimétrica e Massa Específica dos RCD Oriundos de Obras de Edificações Verticais de Maceió. In: ENCONTRO NACIONAL DE TECNOLOGIA DO AMBIENTE CONSTRUÍDO, 13., Canela, 2010. Anais... Canela: ANTAC, 2010.

ASSOCIAÇÃO BRASILEIRA DAS EMPRESAS DE LIMPEZA PÚBLICA E RESÍDUOS ESPECIAIS. Panorama dos Resíduos Sólidos no Brasil 2014. 120 f. 2014. Disponível em: <http://www.abrelpe.org.br/Panorama/panorama20 14.pdf>. Acesso em: 19 jul. 2016.

BERNARDES, A. et al. Quantificação e Classificação dos Resíduos da Construção e Demolição Coletados no Município de Passo Fundo, RS. Ambiente Construído, Porto Alegre, v. 8, n. 3, p. 65-76, jul./out. 2008.

BLUMENSCHEIN, R. N. Manual Técnico: gestão de resíduos sólidos em canteiros de obras. Brasília: Serviço de Apoio às Micro e Pequenas Empresas do Distrito Federal, 2007. Disponível em: <http://bibliotecadigital.puccampinas.edu.br/services/ebooks/Manual\%2520T\%25C3\%25A9cnico\%2520

\%2520Gest\%25C3\%25A3o\%2520de\%2520Res\% 25C3\%25ADduos\%2520S\%25C3\%25B3lidos\%25 20em\%2520Canteiros\%2520de\%2520Obras.pdf>. Acesso em: 19 jul. 2016.

BRASIL. Lei no 12.305, de 2 de agosto de 2010. Brasil. Disponível em:

<http://www.planalto.gov.br/ccivil_03/_ato20072010/2010/lei/l12305.htm>. Acesso em: 27 jun. 2017.
CAETANO, M. O.; SELBACH, J. B. O.; GOMES, L. P. Composição Gravimétrica dos RCD Para a Etapa de Acabamento em Obras Residenciais Horizontais. Ambiente Construído, Porto Alegre, v. 16, n. 2, p. 51-67, abr./jun. 2016.

\section{CONSELHO NACIONAL DO MEIO}

AMBIENTE. Resolução no $\mathbf{3 0 7}$, de 5 de julho de 2002, que estabelece diretrizes, critérios e procedimentos para a gestão dos resíduos da construção civil. Brasília, 2002. Disponível em: <http://www.mma.gov.br/port/conama/legiabre.cf m?codlegi=307>. Acesso em: 7 jan. 2016.

DALTRO FILHO, J. et al. Avaliação da Composição e Quantidade dos Resíduos Sólidos da Construção Civil de Aracaju-Sergipe-Brasil. In: SIMPÓSIO ÍTALO BRASILEIRO DE ENGENHARIA SANITÁRIA E AMBIENTAL, 8., Fortaleza, 2006. Anais... Fortaleza: Associação Brasileira de Engenharia Sanitária e Ambiental, 2006.

DIAS, M. F. Modelo Para Estimar a Geração de Resíduos na Produção de Obras Residenciais Verticais. São Leopoldo, 2013. 117 f. Dissertação (Mestrado em Engenharia Civil) - Programa de Pós-graduação em Engenharia Civil, São Leopoldo, 2013.

DING, T.; XIAO, J. Estimation of BuildingRelated Construction and Demolition Waste in Shanghai. Waste Management, v. 34, p. 2327 2334, 2014.

FORMOSO, C. T. et al. Material Waste in Building Industry: main causes and prevention. Journal of Construction Engineering and Management, v. 128, n. 4, p. 316-325, 2002.

HAIR JUNIOR, J. F. et al. Análise Multivariada de Dados. 5. ed. Porto Alegre: Bookman, 2005.

JOHN, V. M. Reciclagem de Resíduos na Construção Civil:contribuição à metodologia de pesquisa e desenvolvimento. São Paulo, 2000. 113 f. Tese (Livre Docência) - Escola de Engenharia, Universidade de São Paulo, São Paulo, 2000.

KARPINSK, L. A. Gestão Diferenciada de Resíduos da Construção Civil: uma abordagem ambiental. Porto Alegre: ediPUCRS, 2009.

KERN, A. P. et al. Waste Generated in High-Rise Buildings Construction: a quantification model based on statistical multiple regression. Waste Management, v. 39, p. 35-44, 2015.

LLATAS, C. A Model for Quantifying Construction Waste in Projects According to the European Waste List. Waste Management, v. 31, n. 6, p. 1261-1276, 2011. 
MÁLIA, M.; BRITO, J. de; BRAVO, M. Indicadores de Resíduos de Construção e Demolição Para Construções Residenciais Novas. Ambiente Construído, Porto Alegre, v. 11, n. 3, p. 117-130, jul./set. 2011.

MASCARÓ, J. L. O Custo das Decisões Arquitetônicas. 4. ed. Porto Alegre: Masquatro, 2006.

MONTGOMERY, D. C.; RUNGER, G. C. Estatística Aplicada e Probabilidade Para Engenheiros. 2. ed. São Paulo: LTC, 2003.

OLIVEIRA, M. E. D. de. et al. Diagnóstico da Geração e da Composição dos RCD de Fortaleza/CE. Engenharia Sanitária e Ambiental, v. 16, n. 3, 219-224, jul./set. 2011.

PICCHI, F. A. Sistemas de Qualidade: uso em empresas de construção de edifícios. São Paulo, 1993. $461 \mathrm{f}$. Tese (Doutorado em Engenharia Civil) - Escola Politécnica, Universidade de São Paulo, São Paulo, 1993.

PINTO, T. de P. Metodologia Para a Gestão Diferenciada de Resíduos Sólidos da CONSTRUÇÃO urbana. São Paulo, 1999. 218 f. Tese (Doutorado em Engenharia de Construção Civil e Urbana) - Escola Politécnica, Universidade de São Paulo, São Paulo, 1999.

SÁEZ, P. V.; MERINO, M. del R.; PORRASAMORES, C. Estimation of Construction and Demolition Waste Volume Generation in New Residential Buildings in Spain. Waste

Management \& Research, v. 30, n. 2, p. 137146, Feb. 2012.
SANTOS, M. D. F. dos. Técnicas Construtivas em Alvenaria Estrutural: contribuição ao uso. Santa Maria, 1998. 157 f. Dissertação (Mestrado em Engenharia) - Programa de Pós-Graduação em Engenharia Civil, Universidade Federal de Santa Maria, Santa Maria, 1998.

SOUZA, U. E. L. de; DEANA, D. F.

Levantamento do Estado da Arte: consumo de materiais. Tecnologias para construção habitacional mais sustentável - Projeto Finep 2386/04, Documento 2.5, 2007. Disponível em: <http://carbonok.com.br/Downloads/Habitacaomai sSustentavel-D2-5-consumo-materiais.pdf>. Acesso em: 19 jul. 2016.

STEVENSON, W. J. Estatística Aplicada à Administração. São Paulo: Harbra, 1981.

TAVARES, L. de P. M. Levantamento e Análise da Deposição e Destinação dos Resíduos da Construção Civil em Ituiutaba, MG. Uberlândia, 2007. 160 f. Dissertação (Mestrado em Engenharia Civil) - Programa de Pós-Graduação em Engenharia Civil, Faculdade de Engenharia Civil, Universidade Federal de Uberlândia, Uberlândia, 2007.

TESSARO, A. B.; SÁ, J. S.; SCREMIN, L. B. Quantificação e Classificação dos Resíduos Procedentes da Construção Civil e Demolição no Município de Pelotas, RS. Ambiente Construído, Porto Alegre, v. 12, n. 2, p. 121-130, abr./jun. 2012.

\section{Marcelo Oliveira Caetano \\ Programa de Pós-Graduação em Engenharia Civil, Unidade Acadêmica de Pesquisa e Pós Graduação | Universidade do Vale do Rio dos Sinos | Av. Unisinos, 950, Cristo Rei | São Leopoldo - RS - Brasil | CEP 93022-750 | Tel.: (51) 3590-8464 | \\ E-mail: mocaetano@unisinos.br}

\section{Asafe Bonisoni Fagundes}

Laboratório de Saneamento Ambiental | Universidade do Vale do Rio dos Sinos | Tel.: (51) 3591-1122 Ramal 1122 |

E-mail: asafebonisoni@hotmail.com

\section{Luciana Paulo Gomes}

Programa de Pós-Graduação em Engenharia Civil, Unidade Acadêmica de Pesquisa e Pós Graduação | Universidade do Vale do Rio dos Sinos | Tel.: (51) 3590-8464 | E-mail: lugomes@unisinos.br

Revista Ambiente Construído

Associação Nacional de Tecnologia do Ambiente Construído

Av. Osvaldo Aranha, 99 - 3o andar, Centro

Porto Alegre - RS - Brasil

CEP $90035-190$

Telefone: +55 (51) 3308-4084

Fax: +55 (51) 3308-4054

www. seer. ufrgs. br/ ambienteconstruido

E-mail: ambienteconstruido@ufrgs.br 